\title{
Construction of a IncRNA-miRNA-mRNA network to determine the regulatory roles of IncRNAs in psoriasis
}

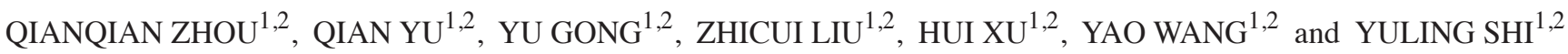 \\ ${ }^{1}$ Department of Dermatology, Shanghai Tenth People's Hospital, Tongji University School of Medicine; \\ ${ }^{2}$ Institute of Psoriasis, Tongji University School of Medicine, Shanghai 200072, P.R. China
}

Received February 17, 2019; Accepted August 14, 2019

DOI: 10.3892/etm.2019.8035

\begin{abstract}
Psoriasis is a chronic inflammatory skin disorder that impairs the quality of life of affected patients. Emerging studies indicate that certain long non-coding RNAs (lncRNAs) have important roles in psoriasis. However, the exact functions of lncRNAs and their regulatory mechanisms as competitive endogenous RNAs (ceRNAs) in psoriasis have remained to be fully elucidated. In the present study, differentially expressed lncRNAs, microRNAs (miRNAs) and mRNAs were identified by analyzing public datasets, and a psoriasis-associated IncRNA-miRNA-mRNA network was constructed based on the ceRNA theory. Furthermore, previously validated abnormally expressed miRNAs in psoriasis were identified by a systematic literature search in the PubMed and Web of Science databases, and a specific miRNA-associated lncRNA-miRNA-mRNA sub-network was extracted. Furthermore, Gene Ontology and Kyoto Encyclopedia of Genes and Genomes pathway enrichment analyses were performed using DAVID 6.8. A total of 253 lncRNAs, 106 miRNAs and 1,156 mRNAs were identified as being differentially expressed between psoriasis skin and healthy control skin. The present study identified two key lncRNAs that may potentially have a role in the pathogenesis of psoriasis: AL035425.3 and Prader Willi/Angelman region RNA 6. This integrative analysis enhances the understanding of the molecular mechanism of psoriasis and may provide novel therapeutic targets for the treatment of psoriasis.
\end{abstract}

\section{Introduction}

Psoriasis is a chronic, systemic, recurrent inflammatory disease, which is characterized by erythema, papules and scaling (1). This condition is caused by genetic and environmental factors, including gene mutations, infection and stress (2).

Correspondence to: Professor Yuling Shi, Department of Dermatology, Shanghai Tenth People's Hospital, Tongji University School of Medicine, Shanghai 200072, P.R. China

E-mail: shiyuling1973@tongji.edu.cn

Key words: network, competing endogenous RNAs, long non-coding RNAs, psoriasis
The pathological manifestations of psoriasis include abnormal keratinocyte differentiation/proliferation and inflammatory cell infiltration (3). It is currently recognized that the pathogenesis of psoriasis is associated with the interleukin (IL)-23/type 17 T-helper cell immune axis, leading to abnormalities in immune cells and associated cytokines, which further induces excessive proliferation of keratinocytes (4). Although extensive research has been performed on the pathogenesis of psoriasis, the mechanisms of psoriasis have remained to be fully elucidated.

Long non-coding RNAs (lncRNAs) are a type of non-coding RNA with a length of $>200 \mathrm{nt}(5,6)$. It is speculated that $\operatorname{lncRNAs}$ are involved in numerous important biological processes, including cellular homeostasis, genomic imprinting, immunity and development (7). In addition, certain lncRNAs have a role in cardiovascular, neurological and developmental diseases, as well as cancers $(5,8)$. Recently, lncRNAs were reported to have pivotal roles in psoriasis. For instance, the lncRNA psoriasis susceptibility-associated RNA gene induced by stress (PRINS) is the most frequent transcript detected in the non-defective epidermis of patients with psoriasis, and it is thought to contribute to the pathogenesis of psoriasis $(9,10)$. Certain studies have reported that PRINS is capable of regulating G1P3, which is expressed at high levels in psoriatic lesions and has an anti-apoptotic role in keratinocytes $(9,11)$. Furthermore, stressors including microbial components and ultraviolet-B radiation are capable of inducing high expression of PRINS in keratinocytes (9). Msh homeobox 2 pseudogene 1 (MSX2P1), which is another lncRNA, facilitates the proliferation of IL-22-stimulated keratinocytes by suppressing microRNA (miRNA/miR)-6731-5p and upregulating the expression of S100A7 (12). In addition, lncRNAs have been indicated to be an important type of competing endogenous RNA (ceRNA) (13). IncRNAs inhibit miRNA-mediated target repression by competing for miRNA-binding sites with mRNAs. However, the regulatory mechanisms involving ceRNAs in psoriasis have remained elusive. In the present study, psoriasis-associated RNA networks were reconstructed based on the ceRNA theory, and the biological functions of the networks were also investigated.

\section{Materials and methods}

Processing of raw data. High-throughput sequencing data for psoriasis-associated lncRNAs, mRNAs and miRNAs 
A

Upregulated lncRNAs

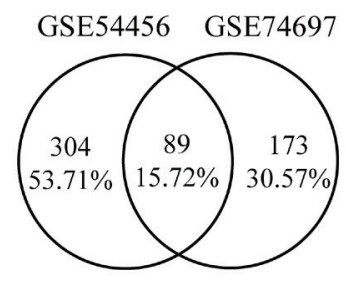

Upregulated mRNAs

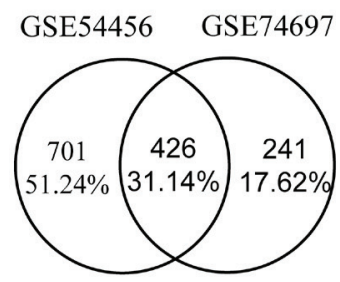

B

miRNAs GSE31037

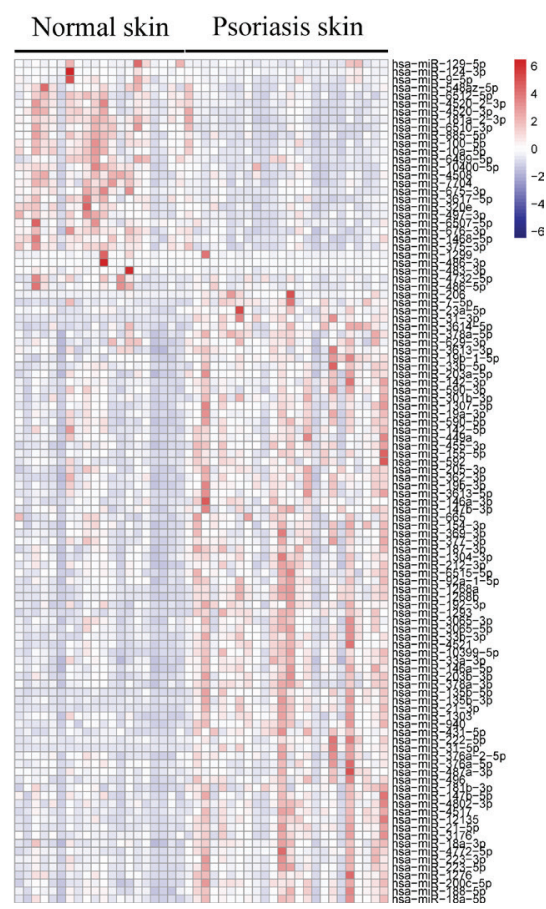

Figure 1. Identification of differentially expressed lncRNAs, mRNAs and miRNAs in psoriasis. (A) Integrated analysis of upregulated (top left) and downregulated (top right) lncRNAs. (B) Integrated analysis of upregulated (bottom left) and downregulated (bottom right) mRNAs. (B) Heat map displaying differential miRNA expression in psoriasis within the GSE31037 dataset. IncRNA, long non-coding RNA; miR/miRNA, microRNA; hsa, Homo sapiens.

were downloaded from the Gene Expression Omnibus (GEO) database (http://www.ncbi.nlm.nih.gov/geo/; lncRNA and mRNA expression profile accession numbers, GSE54456 and GSE74697; miRNA expression profile expression number, GSE31037). For the high-throughput raw sequencing data for lncRNAs and mRNAs, quality assessment was performed with FastQC (v0.11.7) (http://www.bioinformatics.babraham. ac.uk/projects/fastqc/); further, sequencing reads were trimmed by removing adapters and low-quality sequences were trimmed by using Trimmomatic (v0.38) (14). Hisat2 (v2.1.0) (15) was used to map the trimmed reads to the human GRCh38 reference genome (http://useast.ensembl. org/info/data/ftp/index.html), and the HTSeq-count (16) was then used to quantify the genes. Furthermore, microarray data (accession no. GSE13355) for comparing the difference in the expression of IncRNAs were also downloaded from the GEO database. After the probe was set, re-annotation was performed to identify specific lncRNAs. The method of the re-annotation method was performed as previously described by Shen et al (17). For the miRNA high-throughput raw sequencing data, miRDeep2 (18) was used to remove adapters, map the trimmed reads to the human GRCh38 reference genome and quantify the expression of miRNAs.

Screening for differentially expressed lncRNAs, miRNAs and $m R N A s$. The differentially expressed lncRNAs, mRNAs and miRNAs between psoriatic lesions involving the skin and healthy controls were identified with the DESeq2 package (19). The differentially expressed lncRNAs, mRNAs and miRNAs were selected according to the adjusted $\mathrm{P}$-values $(\mathrm{P}<0.05$ and $\mid \log 2$ fold changel $>1)$.
Functional enrichment analysis. DAVID 6.8 (20) was used to perform the Gene Ontology (GO) functional enrichment analysis in the category Biological Processes (BP), and Kyoto Encyclopedia of Genes and Genomes (KEGG) pathway functional enrichment analysis of differentially expressed genes was also performed. $\mathrm{P}<0.05$ was considered to indicate statistical significance.

Construction of the psoriasis-associated IncRNA-miRNAmRNA network. miRNA-RNA interactions were downloaded from the experimentally verified databases DIANA-TarBase V8 (21) and miRTarBase (V7.0) (22), and miRNA-lncRNA interactions were downloaded from starBase v2.0 (23) and LncBase V2 (24). To construct the psoriasis-associated ceRNA network, the differentially expressed miRNAs, mRNAs and lncRNAs in psoriasis vs. normal samples were integrated with the miRNA-mRNA and miRNA-lncRNA pairs downloaded from the databases. Subsequently, miRNA-mRNA and miRNA-lncRNA pairs that shared common miRNAs were integrated into the psoriasis-associated ceRNA network. In the present study, to create the psoriasis-associated ceRNA network, only upregulated lncRNAs and downregulated miRNAs were integrated with upregulated mRNAs, while only downregulated lncRNAs and upregulated miRNAs were integrated with downregulated mRNAs (25). The degree of interaction - a topological property that indicates the number of edges that connect to a node-was also calculated. IncRNAs in the ceRNA network that interacted with >5 different miRNAs were identified as key lncRNAs in the present study. The networks were visualized using Cytoscape 3.6.1 (26). 
A

Biological processes of up-regulated genes

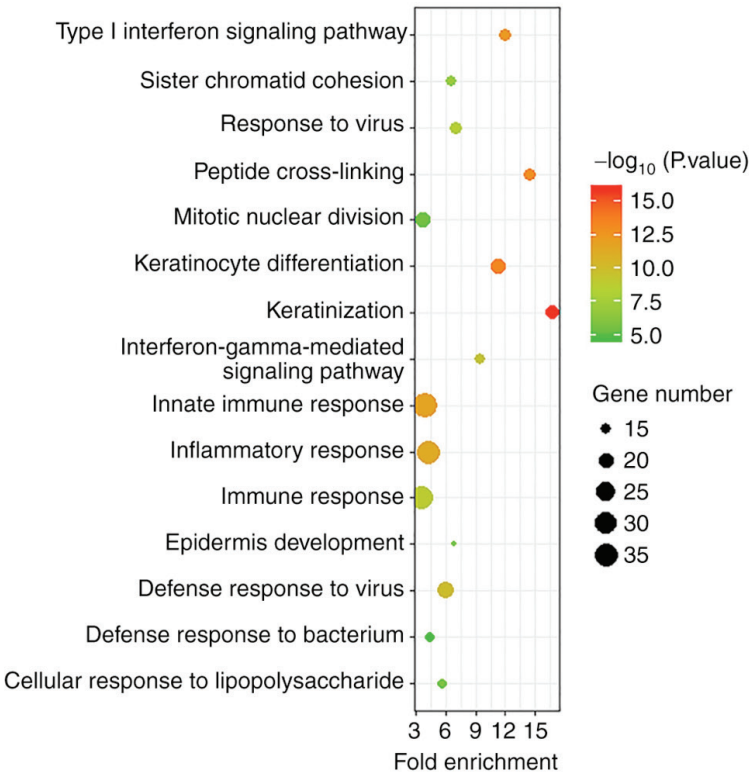

C

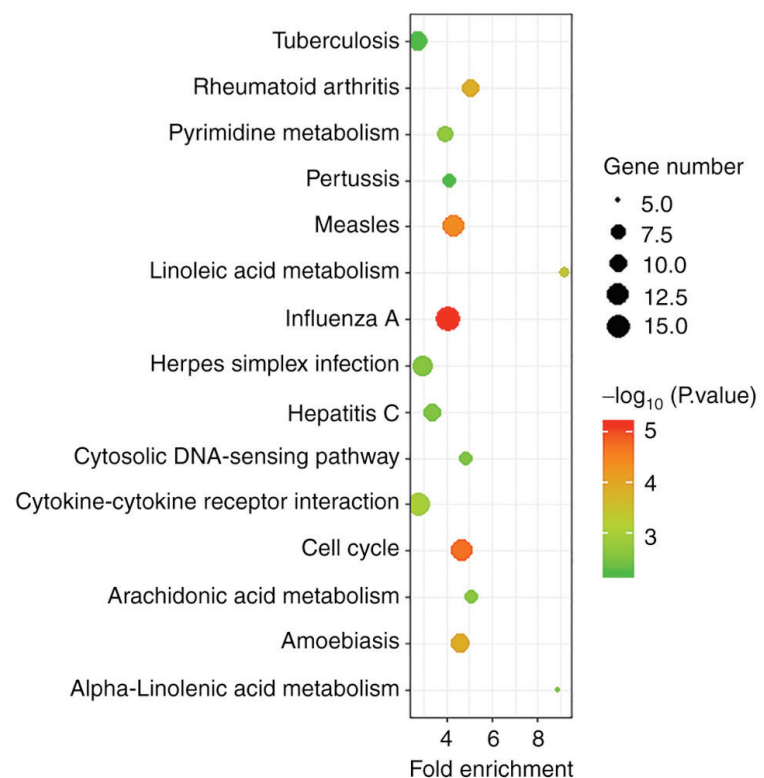

B Biological processes of down-regulated genes

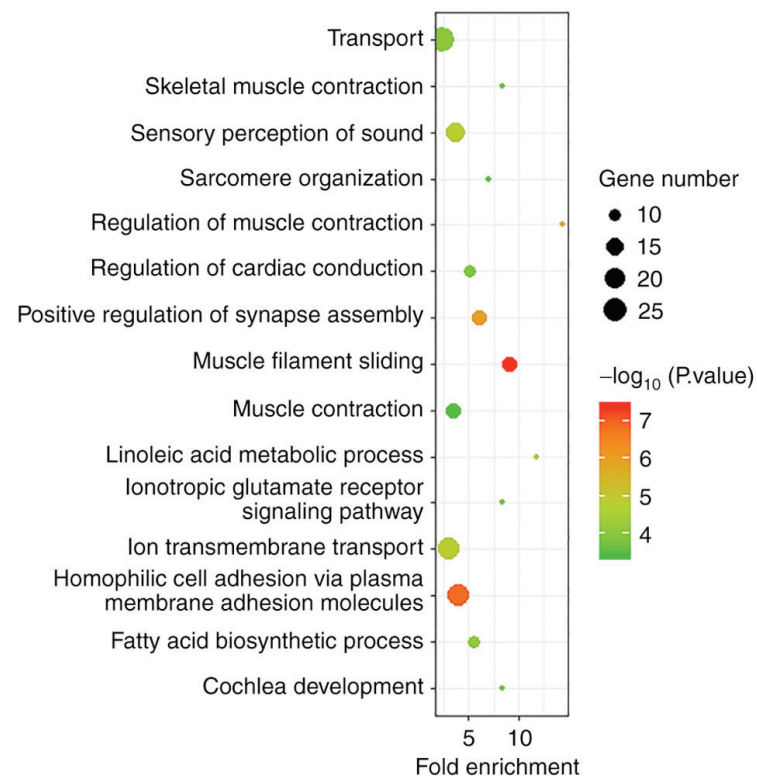

D KEGG pathways of down-regulated genes

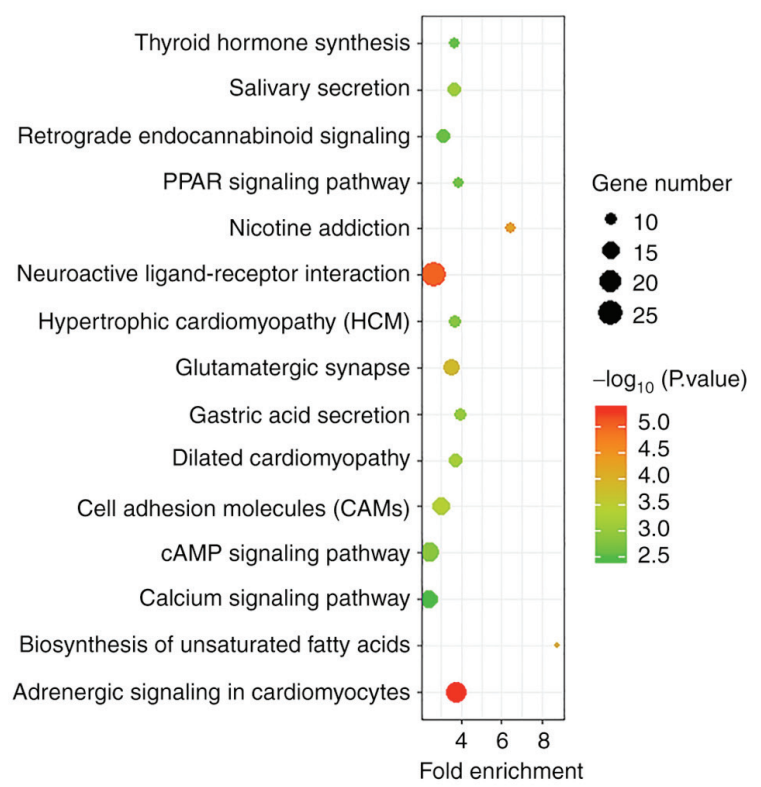

Figure 2. Biological function and pathway analysis of differentially expressed genes (top 15). (A) GO biological process enrichment analysis of upregulated mRNAs. (B) GO biological process enrichment analysis of downregulated mRNAs. (C) KEGG pathway analysis of upregulated mRNAs. (D) KEGG pathway analysis of downregulated mRNAs. KEGG, Kyoto Encyclopedia of Genes and Genomes; GO, gene ontology.

\section{Results}

Identification of differentially expressed lncRNAs, mRNAs and miRNAs. Analysis of the high-throughput data deposited under accession numbers GSE54456 and GSE74697 revealed that a total of 89 upregulated lncRNAs and 164 downregulated lncRNAs were shared between the datasets for psoriatic lesions involving the skin and healthy controls, 426 upregulated mRNAs and 730 downregulated mRNAs were shared between the two datasets (Fig. 1A). The sRNA sequencing data (GSE31037) were obtained from biopsy samples of 24 psoriasis lesions from skin biopsies and 20 normal skin biopsies from healthy controls. The expression profiles of
miRNAs between psoriasis-affected skin and healthy control skin were compared with the R Deseq2 package, and 77 upregulated miRNAs and 29 downregulated miRNAs were identified (Fig. 1B).

Functional enrichment analysis of differentially expressed $m R N A s$ in psoriasis. To investigate the biological mechanisms of IncRNAs in the development of psoriasis, GO functional and KEGG pathway enrichment analyses were performed for differentially expressed genes (DEGs) in psoriasis. The results indicated that the upregulated mRNAs were enriched for the following GO terms: Keratinization, keratinocyte differentiation, peptide cross-linking, type I interferon 


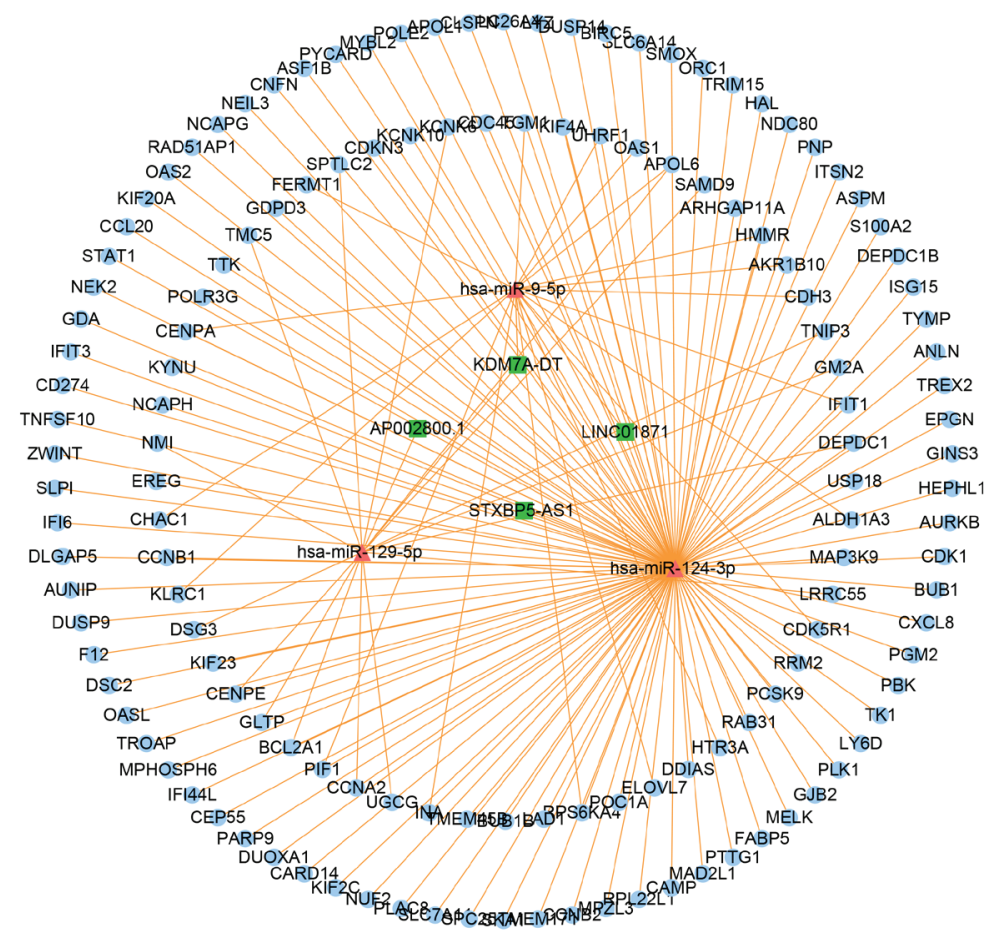

Figure 3. Construction of upregulated lncRNA-mediated ceRNA network. Green nodes, lncRNAs; red nodes, miRNAs; blue nodes, mRNAs. lncRNA, long non-coding RNA; miR/miRNA, microRNA; hsa, Homo sapiens.

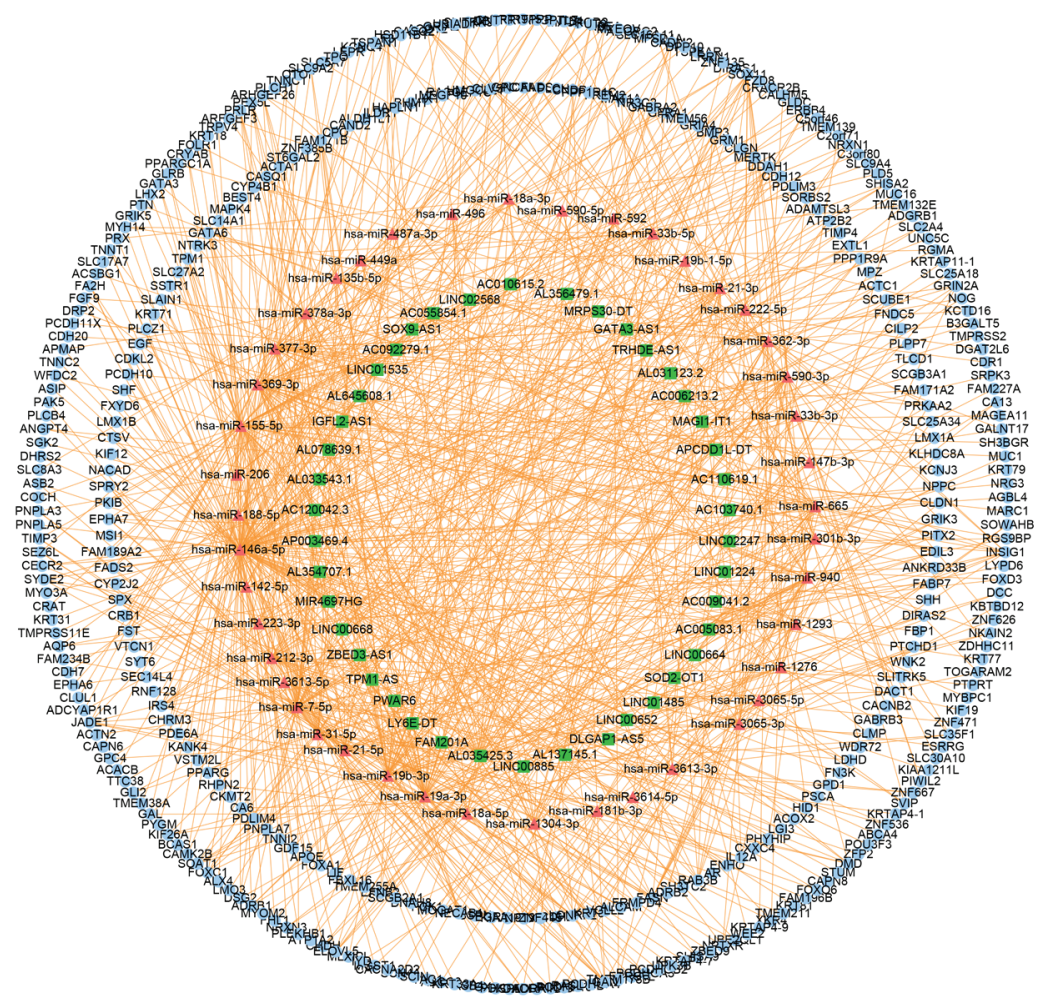

Figure 4. Construction of downregulated lncRNA-mediated ceRNA network. Green nodes, lncRNAs; red nodes, miRNAs; blue nodes, mRNAs. IncRNA, long non-coding RNA; miR/miRNA, microRNA; hsa, Homo sapiens.

signaling pathway, innate immune response, inflammatory response, defence response to virus, interferon- $\gamma$-mediated response, immune response, response to virus, sister chromatid cohesion, epidermis development, cellular response to lipopolysaccharide, mitotic nuclear division and defence response to bacterium (Fig. 2A). By contrast, the downregulated genes were enriched in the following GO terms: Muscle filament sliding, homophilic cell adhesion via 

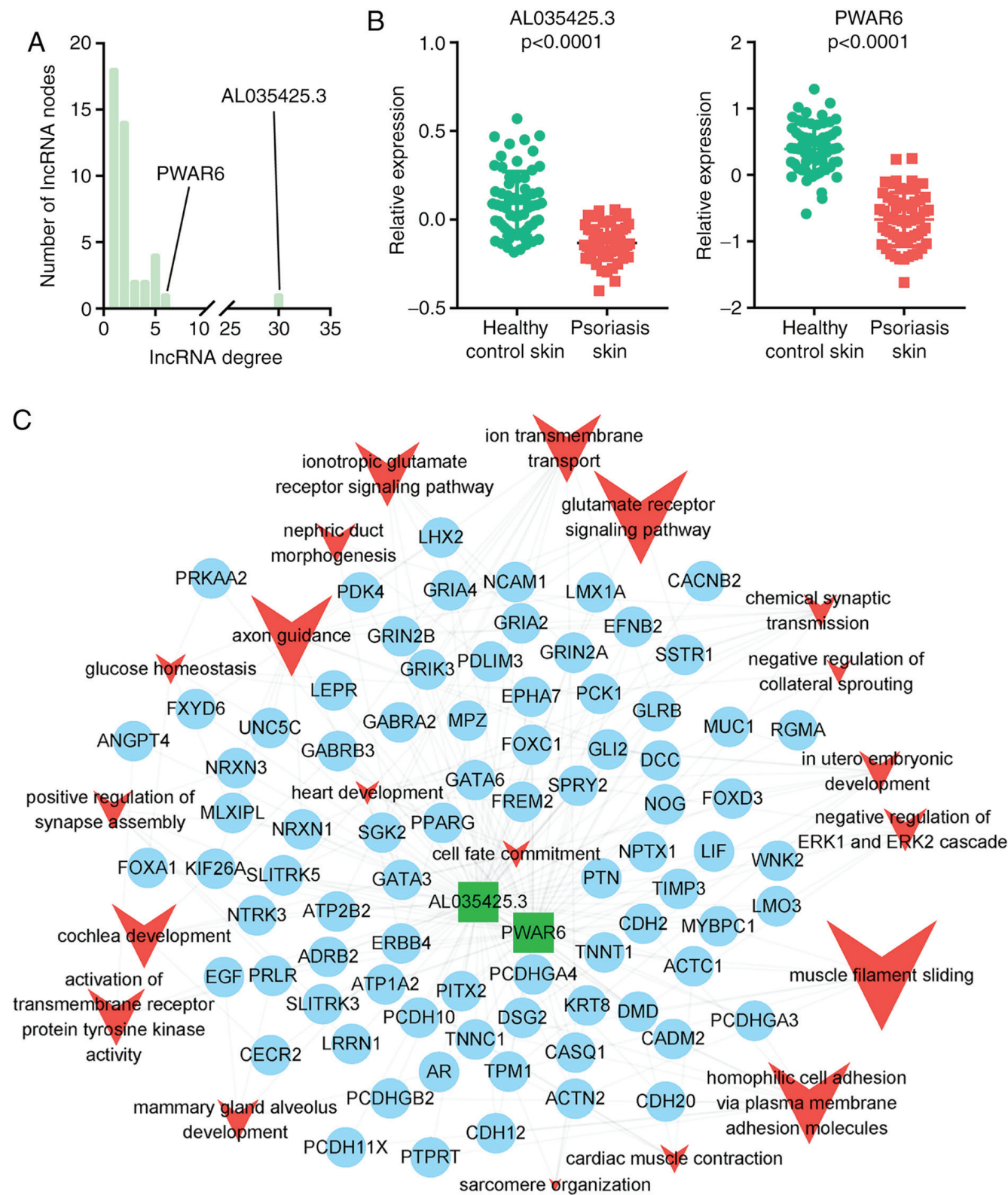

Figure 5. Identification of key lncRNA analysis. (A) The degree distribution of lncRNAs in the downregulated lncRNA-mediated ceRNA network are presented. (B) Expression level of two key lncRNAs in psoriasis skin and healthy control skin. (C) Key downregulated lncRNA-mediated biological processes analysis. lncRNA, long non-coding RNA; ceRNA, competing endogenous RNA; PWAR6, Prader Willi/Angelman region RNA 6.

plasma membrane adhesion molecules, positive regulation of synapse assembly, regulation of muscle contraction, ion transmembrane transport, linoleic acid metabolic process, sensory perception of sound, fatty acid biosynthesis process, transport, regulation of cardiac conduction, cochlear development, ionotropic glutamate receptor signaling pathway, skeletal muscle contraction, muscle contraction and sarcomere organization (Fig. 2B).

The most significant KEGG pathway terms for the upregulated mRNAs are provided in Fig. 2C: Influenza A, cell cycle, measles, amoebiasis, rheumatoid arthritis, linoleic acid metabolism, cytokine-cytokine receptor interaction, pyrimidine metabolism, $\alpha$-linolenic acid metabolism, arachidonic acid metabolism, herpes simplex infection, hepatitis C, cytosolic DNA-sensing pathway, tuberculosis and pertussis. The downregulated genes were mainly involved in adrenergic signaling in cardiomyocytes, neuroactive ligand-receptor interaction, nicotine addiction, biosynthesis of unsaturated fatty acids, glutamatergic synapse, cell adhesion, dilated cardiomyopathy, salivary secretion, gastric acid secretion, cAMP signaling pathway, hypertrophic cardiomyopathy, peroxisome proliferator activated receptor- $\alpha$ signaling pathway, retrograde endocannabinoid signaling, thyroid hormone synthesis and calcium signaling pathway (Fig. 2D).

Construction of the psoriasis-associated ceRNA network. As presented in Figs. 3 and 4, the upregulated IncRNA-mediated ceRNA network contained 4 lncRNA nodes, 3 miRNA nodes and 139 mRNA nodes, and the downregulated lncRNA-mediated ceRNA network contained 42 lncRNA nodes, 43 miRNA nodes and 382 mRNA nodes. 
Identification of key lncRNAs. IncRNA nodes that interacted with $>5$ different miRNAs were identified as key lncRNAs, which may have critical roles in biological networks. Of note, in the downregulated lncRNA-mediated ceRNA network, only two lncRNAs, AL035425.3 and Prader Willi/Angelman region RNA 6 (PWAR6), interacted with $>5$ different miRNAs (Fig. 5A), while in the upregulated lncRNA-mediated ceRNA network, none of the IncRNAs interacted with $>5$ different miRNAs. To further validate the differentially expressed key lncRNAs, a t-test was used to compare their expression between psoriasis lesions involving the skin and healthy controls. As presented in Fig. 5B, the key lncRNAs were downregulated in psoriasis-affected skin. To better understand the mechanism of those key lncRNAs in psoriasis, sub-ceRNA networks were extracted and GO functional enrichment analysis for the DEGs associated with those key lncRNAs was performed (Fig. 5C). In the GO enrichment analysis, the DEGs associated with 1ncRNA AL035425.3 and PWAR6 were involved in the biological processes of muscle filament sliding, glutamate receptor signaling pathway, homophilic cell adhesion via plasma membrane adhesion molecules, axon guidance, ion transmembrane transport, cochlear development and ionotropic glutamate receptor signaling pathway.

Construction of a specific validated miRNA-associated lncRNA-miRNA-mRNA sub-network. A systematic literature search in the PubMed and Web of Science databases was performed to identify real-time PCR-validated differentially expressed microRNAs, using the following key words: 'MicroRNA', 'microRNAs', 'miRNA', 'miRs' and 'Psoriasis'. A total of 37 miRNAs were identified, including 20 downregulated miRNAs and 17 upregulated miRNAs (Table I) (27-48). Of the identified miRNAs, 7 validated upregulated miRNAs were also present in the downregulated lncRNA-mediated ceRNA network, but none of the validated downregulated miRNAs were present in the upregulated lncRNA-mediated ceRNA network (Fig. 6A). The validated differentially expressed miRNA-associated lncRNA-miRNA-mRNA sub-networks were extracted (Fig. 6B). Finally, DEGs were identified from the literature. The miRNAs associated with the IncRNA-miRNA-mRNA sub-network were subsequently extracted and functional enrichment analysis was performed (Tables II and III). These genes were mainly enriched in the following GO terms: Muscle filament sliding, regulation of muscle contraction, in utero embryonic development, and enriched in the following KEGG pathways: AMPK signalling pathway, neuroactive ligand-receptor interaction, hypertrophic cardiomyopathy, calcium signalling pathway, insulin signalling pathway, adipocytokine signalling pathway, dilated cardiomyopathy, adrenergic signalling in cardiomyocytes and insulin resistance.

\section{Discussion}

Psoriasis is a chronic immune-mediated inflammatory disease that affects $3.2 \%$ of the adult population in the US (49). Over the past decades, the role of different cytokines in psoriasis has been studied widely (50), and therefore, cytokine-targeting drugs have been developed, including adalimumab (which targets tumor necrosis factor- $\alpha$ ), secukinumab (which targets IL-17A) and ustekinumab (which targets IL-12 and IL-23
Table I. Differentially expressed miRNAs in psoriasis skin identified by the literature search.

A, Downregulated miRNAs

\begin{tabular}{|c|c|c|c|}
\hline \multirow[b]{2}{*}{ miRNA name } & \multicolumn{2}{|c|}{$\begin{array}{l}\text { miRNA fold-change } \\
\text { in GSE31037 }\end{array}$} & \multirow[b]{2}{*}{ (Refs.) } \\
\hline & $\log 2$ fold-change & P-value & \\
\hline miR-10b-5p & -0.84 & $2.27 \times 10^{-5}$ & (27) \\
\hline $\operatorname{miR}-125 b$ & -0.91 & $1.12 \times 10^{-15}$ & (28) \\
\hline miR-138 & -0.22 & $2.98 \times 10^{-2}$ & (29) \\
\hline miR-143-3p & -0.33 & $4.32 \times 10^{-2}$ & (27) \\
\hline miR-145-5p & -0.05 & $7.84 \times 10^{-1}$ & (27) \\
\hline miR-181b-5p & -0.58 & $9.74 \times 10^{-3}$ & $(30)$ \\
\hline miR-194 & -0.28 & $1.17 \times 10^{-1}$ & (31) \\
\hline miR-196b-5p & -0.62 & $4.00 \times 10^{-5}$ & (27) \\
\hline miR-197 & -0.28 & $2.11 \times 10^{-2}$ & (32) \\
\hline $\operatorname{miR}-20 a-3 p$ & 0.61 & $1.26 \times 10^{-4}$ & (33) \\
\hline miR-217 & 0.17 & $5.51 \times 10^{-1}$ & (34) \\
\hline miR-320b & -0.54 & $5.42 \times 10^{-4}$ & (35) \\
\hline $\operatorname{miR}-338-3 p$ & -0.48 & $5.24 \times 10^{-3}$ & (27) \\
\hline miR-423 & 0.05 & $8.52 \times 10^{-1}$ & (32) \\
\hline $\operatorname{miR}-424$ & 0.83 & $1.99 \times 10^{-6}$ & (36) \\
\hline $\operatorname{miR}-4516$ & -0.90 & $1.03 \times 10^{-2}$ & (37) \\
\hline miR-486-3p & -1.09 & $4.17 \times 10^{-3}$ & (38) \\
\hline miR-876-5p & 0.06 & $9.37 \times 10^{-1}$ & (39) \\
\hline miR-99a & -0.99 & $2.47 \times 10^{-11}$ & (32) \\
\hline $\operatorname{miR}-675-3 p$ & -2.05 & $2.56 \times 10^{-11}$ & $(40)$ \\
\hline
\end{tabular}

B, Upregulated miRNAs

\section{miRNA fold-change in GSE31037}

\begin{tabular}{lcll}
\cline { 2 - 3 } miRNA name & $\log 2$ fold-change & P-value & (Refs.) \\
\hline miR-122-5p & -0.48 & $6.17 \times 10^{-1}$ & $(41)$ \\
miR-130a-3p & 0.01 & $9.55 \times 10^{-1}$ & $(42)$ \\
miR-135b-5p & 2.56 & $2.46 \times 10^{-33}$ & $(40)$ \\
miR-146a-5p & 1.28 & $1.67 \times 10^{-12}$ & $(27)$ \\
miR-146b & 0.36 & $9.03 \times 10^{-2}$ & $(43)$ \\
miR-155-5p & 1.53 & $1.60 \times 10^{-12}$ & $(44)$ \\
miR-203 & -0.12 & $5.77 \times 10^{-1}$ & $(28)$ \\
miR-205 & 0.56 & $8.04 \times 10^{-5}$ & $(45)$ \\
miR-21-5p & 2.07 & $2.40 \times 10^{-26}$ & $(27)$ \\
miR-210 & -0.54 & $2.03 \times 10^{-2}$ & $(46)$ \\
miR-221 & -0.07 & $6.02 \times 10^{-1}$ & $(45)$ \\
miR-222-3p & 0.11 & $4.26 \times 10^{-1}$ & $(45)$ \\
miR-31-5p & 5.46 & $7.08 \times 10^{-49}$ & $(27)$ \\
miR-3613-5p & 1.68 & $2.16 \times 10^{-11}$ & $(40)$ \\
miR-369-3p & 1.30 & $3.78 \times 10^{-11}$ & $(47)$ \\
miR-431 & 2.20 & $1.67 \times 10^{-7}$ & $(40)$ \\
miR-744-3p & 0.38 & $2.78 \times 10^{-2}$ & $(40)$ \\
\end{tabular}

miRNA/miR, microRNA. 
A Confirmed upregulated miRNAs

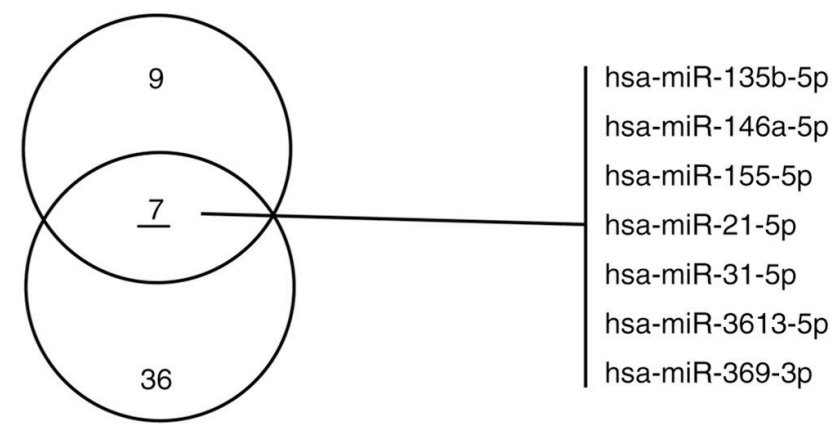

miRNAs from downregulated IncRNA mediated ceRNA network

$\mathrm{B}$

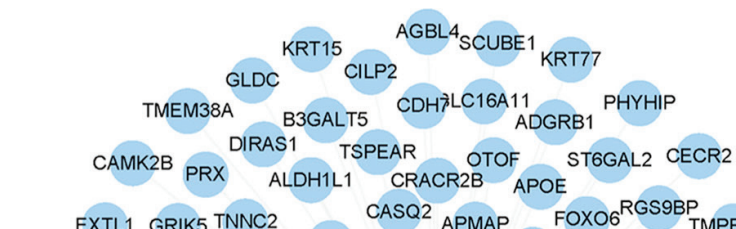

EXTL1 GRIK5 TNNC2 CADHGA3 APMAP FOXO6 RGS9BP TMPRSS2

TRPV4 STUM KRT19 ABCC8MAPK4 ADRA2B RHPN2 ACOX2

TRPV4 STUM KRT19 ABCC8MAPK4 ${ }_{\text {FADS2 }}^{\text {AGI3 }} \begin{aligned} & \text { A ACOX2 } \\ & \text { LINC02568 }\end{aligned}$

SLC9A2 ABCA4 ASIP TMPRSS11E CTSV KIF19 SEC14L4 FOXD3

PLEKHB1 GALNT17 SCGB2A1 FLCH2 FAN CAPN8 LDHD
RASGEF1C

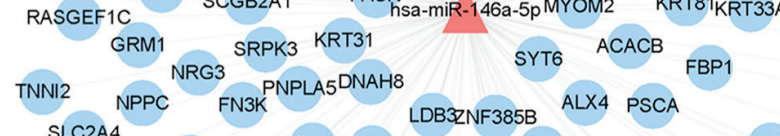

SLC2A4 NACAD SHF

ANGPTL7 MUC16 CKMT2 NACAD SHF TPO SHH KIAA1211L

FXYD6 MS11 PYGM ASB2 PDE6AMYH14 ENHO TIMP4 KRT8 GDF15

TPPP KIF12 ${ }_{\text {SCGB } 3 A 1}$ ACTA1 ${ }_{\text {DHRS2 }}^{\text {CHDH }}$ CACNA2D2

LMX1B ${ }_{\text {ZNF536 POUIF }}^{\text {SCGB3A1 }}{ }_{\text {POUF }}$ CACNA2D2

BEST4 ${ }^{\text {SYDE2 }}$ MIR4697HG TMEM56

AC110619.1 AC103740.1

AL078639.1 AL645608.1 $\quad$ ERBB4 $\underset{\text { NRXN1 }}{\text { SSTR }}$

\begin{tabular}{cccc} 
& \multicolumn{2}{c}{ MYEOV CLUL1 } \\
CRYAB & AC010615.2 & \multicolumn{1}{c}{ SLC27A2 } \\
ANKRD33B CLGN DACT1 & FAM189A2
\end{tabular}

WFDC2 TMEM139 GLRB MGST1 CRB1 FADS1 SH3BGR

$\begin{array}{lll}\text { VSTM2L NOG UBE2QL1FHL1 ADRB2 } & \end{array}$

F3 FAM171A2 DSG2 PDK4 KRT18 PKIB PTN

FOLR1 hsa-miR-155-5p CLDN1 GAL MARC1 EPHA7 FAM201A FGF9 MAGEA11 ${ }_{\text {C5orf46 }}^{\text {FGF9 }} \underset{\text { KANK4 }}{\text { RAB3B }}{ }_{\mathrm{CDH}}^{\mathrm{COCH}}$

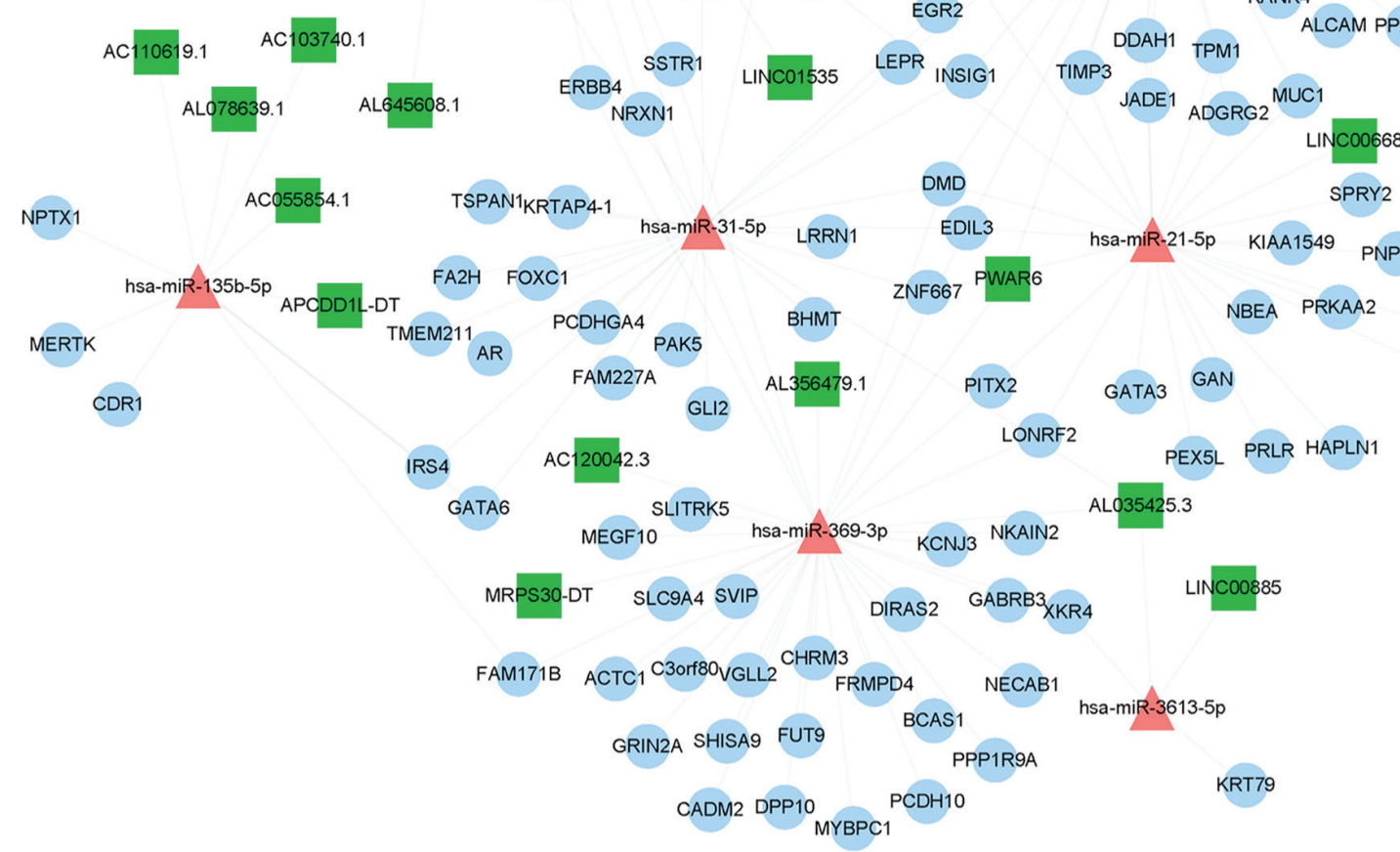

Figure 6. Construction of a specific literature confirmed miRNA-associated lncRNA-miRNA-mRNA sub-network. (A) Intersection of confirmed upregulated miRNAs and miRNAs from downregulated lncRNA-mediated ceRNA network. (B) Confirmed differentially expressed miRNA-associated IncRNA-miRNA-mRNA sub-network. Green nodes, lncRNAs; red nodes, miRNAs; blue nodes, mRNAs. lncRNA, long non-coding RNA; miR/miRNA, microRNA; hsa, Homo sapiens. 
Table II. Gene Ontology enrichment analysis of differentially expressed mRNAs from the specific miRNA-associated long non-coding RNA-miRNA-mRNA sub-networks (top 20).

\begin{tabular}{|c|c|c|c|}
\hline Term & $\mathrm{n}(\%)$ & P-value & Genes \\
\hline GO:0030049 muscle filament sliding & $9(3.93)$ & $1.17 \times 10^{-8}$ & $\begin{array}{l}\text { ACTC1, TNNT1, TNNC2, ACTA1, TNNC1, } \\
\text { MYBPC1, DMD, TPM1, TNNI2 }\end{array}$ \\
\hline GO:0006937 regulation of muscle contraction & $5(2.18)$ & $1.71 \times 10^{-5}$ & TNNT1, TNNC2, TNNC1, TPM1, TNNI2 \\
\hline GO:0001701 in utero embryonic development & $11(4.80)$ & $7.68 \times 10^{-5}$ & $\begin{array}{l}\text { MUC1, AR, NOG, LMX1B, GATA6, GATA3, } \\
\text { FOXC1, GLI2, TPM1, PITX2, FOXD3 }\end{array}$ \\
\hline GO:0003009 skeletal muscle contraction & $5(2.18)$ & $1.66 \times 10^{-4}$ & TNNT1, TNNC2, TNNC1, MYH14, TNNI2 \\
\hline GO:0060048 cardiac muscle contraction & $6(2.62)$ & $1.81 \times 10^{-4}$ & ACTC1, TNNC1, DMD, TPM1, CASQ2, TNNI2 \\
\hline GO:0045214 sarcomere organization & $5(2.18)$ & $3.54 \times 10^{-4}$ & KRT19, KRT8, LDB3, TPM1, CASQ2 \\
\hline GO:0007267 cell-cell signaling & $11(4.80)$ & $8.84 \times 10^{-4}$ & $\begin{array}{l}\text { AR, ADRB2, CRB1, SSTR1, FGF9, FADS1, } \\
\text { ADRA2B, GDF15, MERTK, ASIP, SHH }\end{array}$ \\
\hline GO:0042493 response to drug & $12(5.24)$ & $9.89 \times 10^{-4}$ & $\begin{array}{l}\text { ACTC1, GATA6, GATA3, PPARG, NPPC, } \\
\text { GRIN2A, PTN, TIMP4, ACACB, GAL, ABCC } 8 \text {, } \\
\text { MGST1 }\end{array}$ \\
\hline GO:0048645 organ formation & $3(1.31)$ & $1.35 \times 10^{-3}$ & AR, GATA6, SHH \\
\hline GO:0045165 cell fate commitment & $5(2.18)$ & $2.08 \times 10^{-3}$ & SPRY2, ERBB4, GATA6, GATA3, PPARG \\
\hline $\begin{array}{l}\text { GO:0007156 homophilic cell adhesion via } \\
\text { plasma membrane adhesion molecules }\end{array}$ & $8(3.49)$ & $2.72 \times 10^{-3}$ & $\begin{array}{l}\mathrm{CDH} 12, \mathrm{CDH} 7, \mathrm{DSG} 2, \mathrm{CADM} 2, \mathrm{PCDH} 10, \\
\mathrm{CDH} 2, \mathrm{PCDHGA} 4, \mathrm{PCDHGA} 3\end{array}$ \\
\hline GO:0007010 cytoskeleton organization & $8(3.49)$ & $3.03 \times 10^{-3}$ & $\begin{array}{l}\text { APOE, DMD, KRT15, PAK5, KRT31, CECR2, } \\
\text { GAN, TPM1 }\end{array}$ \\
\hline GO:0042472 inner ear morphogenesis & $5(2.18)$ & $3.26 \times 10^{-3}$ & SPRY2, KCNQ4, FGF9, GATA3, INSIG1 \\
\hline GO:0007605 sensory perception of sound & $7(3.06)$ & $4.96 \times 10^{-3}$ & $\begin{array}{l}\text { COCH, SPRY2, KCNQ4, TSPEAR, GABRB3, } \\
\text { OTOF, MYH14 }\end{array}$ \\
\hline GO:0009953 dorsal/ventral pattern formation & $4(1.75)$ & $6.22 \times 10^{-3}$ & NOG, LMX1B, LHX2, SHH \\
\hline GO:0042593 glucose homeostasis & $6(2.62)$ & $6.89 \times 10^{-3}$ & $\begin{array}{l}\text { SLC2A4, LEPR, PDK4, PPARG, TRPV4, } \\
\text { PRKAA2 }\end{array}$ \\
\hline GO:0043627 response to estrogen & $5(2.18)$ & $7.25 \times 10^{-3}$ & KRT19, GATA6, GATA3, PPARG, GAL \\
\hline GO:0032868 response to insulin & $5(2.18)$ & $8.06 \times 10^{-3}$ & EGR2, FADS1, TRPV4, GAL, ABCC8 \\
\hline $\begin{array}{l}\text { GO:0007171 activation of transmembrane } \\
\text { receptor protein tyrosine kinase activity }\end{array}$ & $3(1.31)$ & $8.45 \times 10^{-3}$ & ADRB2, NRG3, PRLR \\
\hline GO:0034332 adherens junction organization & $4(1.75)$ & $9.35 \times 10^{-3}$ & $\mathrm{CDH} 12, \mathrm{CDH} 7, \mathrm{CADM} 2, \mathrm{CDH} 2$ \\
\hline
\end{tabular}

GO, Gene Ontology; miRNA, microRNA.

p40) (51). However, the roles of IncRNAs in psoriasis have remained largely elusive.

lncRNAs have important roles in immune and inflammatory pathways by regulating gene expression through multiple mechanisms (52). Among these mechanisms, the ceRNA theory is commonly studied. According to this theory, lncRNAs act as 'sponges' for miRNAs and decrease the effects of miRNAs on their target genes, thus promoting the expression of target genes (53). For instance, Qiao et al (12) reported that lncRNA-MSX2P1 activates S100A71 and facilitates the growth of IL-22-stimulated keratinocytes by inhibiting miR-6731-5p. Furthermore, Li et al (54) demonstrated that IncRNA H19 regulates the differentiation of keratinocytes by increasing desmoglein 1 expression through sponging miR-130b-3p.

In the present study, psoriasis-associated lncRNA-miRNAmRNA networks were constructed based on the ceRNA theory, and the DEGs were also subjected to GO BP enrichment analysis and KEGG pathway enrichment analysis.
GO BP enrichment analysis of the upregulated mRNAs indicated that the significantly enriched GO BP terms were psoriasis-associated biological processes, including keratinization (55), keratinocyte differentiation (3), inflammatory response (56) and immune response (56). Furthermore, the downregulated mRNAs were mainly enriched in the BP terms muscle filament sliding, homophilic cell adhesion via plasma membrane adhesion molecules, positive regulation of synapse assembly and regulation of muscle contraction.

In the present study, the downregulated IncRNAs AL035425.3 and PWAR6 were identified as key lncRNAs. GO BP analysis of the key lncRNAs revealed that AL035425.3 and PWAR6 were involved in muscle filament sliding, the glutamate receptor signaling pathway, homophilic cell adhesion via plasma membrane adhesion molecules, axon guidance, ion transmembrane transport, cochlear development and ionotropic glutamate receptor signaling pathway. Of the two key lncRNAs, PWAR6 was previously reported 
Table III. Kyoto Encyclopedia of Genes and Genomes signaling pathway enrichment analysis of differentially expressed mRNAs from the specific miRNAs-associated long non-coding RNA-miRNA-mRNA sub-network.

\begin{tabular}{|c|c|c|c|}
\hline Term & $\mathrm{n}(\%)$ & P-value & Genes \\
\hline hsa04152:AMPK signaling pathway & $8(3.49)$ & $8.22 \times 10^{-4}$ & $\begin{array}{l}\text { IRS4, SLC2A4, LEPR, PPARG, FASN, FBP1, } \\
\text { PRKAA2, ACACB }\end{array}$ \\
\hline $\begin{array}{l}\text { hsa04080:Neuroactive ligand-receptor } \\
\text { interaction }\end{array}$ & $11(4.80)$ & $2.25 \times 10^{-3}$ & $\begin{array}{l}\text { GLRB, ADRB2, PRLR, GABRB3, CHRM3, SSTR1, } \\
\text { LEPR, GRIK5, GRIN2A, ADRA2B, GRM1 }\end{array}$ \\
\hline hsa05410:Hypertrophic cardiomyopathy & $6(2.62)$ & $2.80 \times 10^{-3}$ & $\begin{array}{l}\text { ACTC1, TNNC1, DMD, PRKAA2, CACNA2D2, } \\
\text { TPM1 }\end{array}$ \\
\hline hsa04020:Calcium signaling pathway & $8(3.49)$ & $6.85 \times 10^{-3}$ & $\begin{array}{l}\text { ADRB2, TNNC2, CHRM3, ERBB4, TNNC1, } \\
\text { GRIN2A, CAMK2B, GRM1 }\end{array}$ \\
\hline hsa04910:Insulin signaling pathway & $7(3.06)$ & $7.39 \times 10^{-3}$ & $\begin{array}{l}\text { IRS4, PYGM, SLC2A4, FASN, FBP1, PRKAA2, } \\
\text { ACACB }\end{array}$ \\
\hline hsa04920:Adipocytokine signaling pathway & $5(2.18)$ & $1.12 \times 10^{-2}$ & IRS4, SLC2A4, LEPR, PRKAA2, ACACB \\
\hline hsa05414:Dilated cardiomyopathy & $5(2.18)$ & $2.06 \times 10^{-2}$ & ACTC1, TNNC1, DMD, CACNA2D2, TPM1 \\
\hline $\begin{array}{l}\text { hsa04261:Adrenergic signaling in } \\
\text { cardiomyocytes }\end{array}$ & $6(2.62)$ & $2.88 \times 10^{-2}$ & $\begin{array}{l}\text { ACTC1, ADRB2, TNNC1, CAMK2B, CACNA2D2, } \\
\text { TPM1 }\end{array}$ \\
\hline hsa04931:Insulin resistance & $5(2.18)$ & $4.58 \times 10^{-2}$ & PYGM, SLC2A4, PRKAA2, ACACB, SLC27A2 \\
\hline
\end{tabular}

miRNA, microRNA; hsa, Homo sapiens.

to be a tumor suppressor lncRNA in glioma, and high expression of PWAR6 was reported to be an indicator of better survival in glioma patients (57). In addition, PWAR6 is functionally important in Prader-Willi syndrome, and the disruption of its expression is associated with the pathogenesis of the disease (58). Two potential target miRNAs of PWAR6 were identified from the validated miRNA-associated IncRNA-miRNA-mRNA sub-network: miR-155-5p and miR-369-3p. Previous studies have demonstrated that knockdown of miR-155-5p suppresses psoriasis-associated inflammatory responses through regulation of the NLR family pyrin domain containing 3 inflammasome (44); furthermore, the expression levels of miR-369-3p in skin had a positive correlation with the Psoriasis Area and Severity Index, which is used to assess disease severity (47). Taken together, these results indicate that PWAR6 may be involved in the progress of psoriasis via regulation of the expression of miR-155-5p and miR-369-3p.

The present study has certain limitations that should be considered: First, although the expression profiles of lncRNA and mRNA were determined from the same specimens, the miRNA expression profile is from an independent dataset. Thus, combining these profiles into a network may introduce selection bias, and further experiments using the same specimens are required. In addition, further validation of key lncRNA expression levels in psoriasis samples is required. Finally, additional functional investigations of these lncRNAs in the context of psoriasis progression are required.

In summary, the present study presented a novel and important psoriasis-associated ceRNA network based on the ceRNA theory. The results indicate that certain lncRNAs have key roles in the development of psoriasis. In addition, the present study identified two lncRNAs, AL035425.3 and PWAR6, that were indicated to have a central role in psoriasis. The present study provides insight into the molecular mechanisms of psoriasis, and this may help to identify novel therapeutic targets for the treatment of psoriasis in the future.

\section{Acknowledgements}

Not applicable.

\section{Funding}

This work was supported by grants from the National Natural Science Foundation of China (grant nos. 81673050 and 81872522), the Program of Science and Technology Commission of Shanghai Municipality (grant no. 18140901800) and the Excellent Subject Leader Program of Shanghai Municipal Commission of Health and Family Planning (grant no. 2018BR30).

\section{Availability of data and materials}

The datasets used and/or analyzed during the current study are available from the corresponding author on reasonable request.

\section{Authors' contributions}

QZ and YS designed the current study, performed data analysis and wrote the manuscript. QY and YG performed the literature review regarding microRNAs in psoriasis. ZL, HX and YW analyzed the data. All authors read and approved the final manuscript.

\section{Ethics approval and consent to participate}

Not applicable. 


\section{Patient consent for publication}

Not applicable.

\section{Competing interests}

The authors declare that they have no competing interests.

\section{References}

1. Nestle FO, Kaplan DH and Barker J: Psoriasis. N Engl J Med 361: 496-509, 2009.

2. Greb JE, Goldminz AM, Elder JT, Lebwohl MG, Gladman DD, Wu JJ, Mehta NN, Finlay AY and Gottlieb AB: Psoriasis. Nat Rev Dis Primers 2: 16082, 2016.

3. Ogawa E, Sato Y, Minagawa A and Okuyama R: Pathogenesis of psoriasis and development of treatment. J Dermatol 45: 264-272, 2018.

4. Di Cesare A, Di Meglio P and Nestle FO: The IL-23/Th17 Axis in the Immunopathogenesis of Psoriasis. J Invest Dermatol 129: 1339-1350, 2009

5. Esteller M: Non-coding RNAs in human disease. Nat Rev Genet 12: 861-874, 2011.

6. ENCODE Project Consortium: An integrated encyclopedia of DNA elements in the human genome. Nature 489: 57-74, 2012.

7. Mercer TR, Dinger ME and Mattick JS: Long non-coding RNAs: Insights into functions. Nat Rev Genet 10: 155-159, 2009.

8. Batista PJ and Chang HY: Long noncoding RNAs: cellular address codes in development and disease. Cell 152: 1298-1307, 2013.

9. Szell M, Danis J, Bata-Csorgo Z and Kemeny L: PRINS, a primate-specific long non-coding RNA, plays a role in the keratinocyte stress response and psoriasis pathogenesis. Pflugers Arch 468: 935-943, 2016.

10. Danis J, Goblos A, Bata-Csorgo Z, Kemeny L and Szell M PRINS Non-Coding RNA regulates nucleic acid-induced innate immune responses of human keratinocytes. Front Immunol 8: 1053, 2017.

11. Szegedi K, Sonkoly E, Nagy N, Németh IB, Bata-Csörgo Z, Kemény L, Dobozy A and Széll M: The anti-apoptotic protein G1P3 is overexpressed in psoriasis and regulated by the non-coding RNA, PRINS. Exp Dermatol 19: 269-278, 2010.

12. Qiao M, Li R, Zhao X, Yan J and Sun Q: Up-regulated IncRNA-MSX2P1 promotes the growth of IL-22-stimulated keratinocytes by inhibiting miR-6731-5p and activating S100A7. Exp Cell Res 363: 243-254, 2018.

13. Salmena L, Poliseno L, Tay Y, Kats L and Pandolfi PP: A ceRNA hypothesis: the Rosetta Stone of a hidden RNA language? Cell 146: 353-358, 2011

14. Bolger AM, Marc L and Bjoern U: Trimmomatic: A flexible trimmer for Illumina sequence data. Bioinformatics 30 2114-2120, 2014

15. Daehwan K, Ben L and Salzberg SL: HISAT: A fast spliced aligner with low memory requirements. Nat Methods 12: 357-360, 2015.

16. Simon A, Paul Theodor P and Wolfgang H: HTSeq-a Python framework to work with high-throughput sequencing data. Bioinformatics 31: 166-169, 2015.

17. Shen L, Liu W, Cui J, Li J and Li C: Analysis of long non-coding RNA expression profiles in ovarian cancer. Oncol Lett 14 1526-1530, 2017

18. Friedl?Nder MR, Mackowiak SD, Na L, Wei C and Nikolaus R miRDeep2 accurately identifies known and hundreds of nove microRNA genes in seven animal clades. Nucleic Acids Res 40: 37-52, 2012 .

19. Love MI, Huber W and Anders S: Moderated estimation of fold change and dispersion for RNA-seq data with DESeq2. Genome Biol 15: 550, 2014.

20. Huang da W, Sherman BT and Lempicki RA: Systematic and integrative analysis of large gene lists using DAVID bioinformatics resources. Nat Protoc 4: 44-57, 2009.

21. Karagkouni D, Paraskevopoulou MD, Chatzopoulos S, Vlachos IS, Tastsoglou S, Kanellos I, Papadimitriou D, Kavakiotis I, Maniou S, Skoufos G, et al: DIANA-TarBase v8: A decade-long collection of experimentally supported miRNA-gene interactions. Nucleic Acids Res 46: D239-D245, 2018.
22. Chou CH, Shrestha S, Yang CD, Chang NW, Lin YL, Liao KW, Huang WC, Sun TH, Tu SJ, Lee WH, et al: miRTarBase update 2018: A resource for experimentally validated microRNA-target interactions. Nucleic Acids Res 46: D296-d302, 2018.

23. Li JH, Liu S, Zhou H, Qu LH and Yang JH: starBase v2.0: decoding miRNA-ceRNA, miRNA-ncRNA and protein-RNA interaction networks from large-scale CLIP-Seq data. Nucleic Acids Res 42: D92-D97, 2014.

24. Paraskevopoulou MD, Vlachos IS, Karagkouni D, Georgakilas G, Kanellos I, Vergoulis T, Zagganas K, Tsanakas P, Floros E, Dalamagas $\mathrm{T}$ and Hatzigeorgiou AG: DIANA-LncBase v2: Indexing microRNA targets on non-coding transcripts. Nucleic Acids Res 44: D231-D238, 2016.

25. Tian L, Hu X, He Y, Wu Z, Li D and Zhang H: Construction of IncRNA-miRNA-mRNA networks reveals functional lncRNAs in abdominal aortic aneurysm. Exp Ther Med 16: 3978-3986, 2018.

26. Shannon P, Markiel A, Ozier O, Baliga NS, Wang JT, Ramage D, Amin N, Schwikowski B and Ideker T: Cytoscape: A software environment for integrated models of biomolecular interaction networks. Genome Res 13: 2498-2504, 2003.

27. Yan JJ, Qiao M, Li RH, Zhao XT, Wang XY and Sun Q: Downregulation of miR-145-5p contributes to hyperproliferation of keratinocytes and skin inflammation in psoriasis. Br J Dermatol 180: 365-372, 2018.

28. Sonkoly E, Wei T, Janson PC, Sääf A, Lundeberg L, Tengvall-Linder M, Norstedt G, Alenius H, Homey B, Scheynius A, et al: MicroRNAs: Novel regulators involved in the pathogenesis of psoriasis? PLoS One 2: e610, 2007.

29. Fu D, Yu W, Li M, Wang H, Liu D, Song X, Li Z and Tian Z MicroRNA-138 regulates the balance of Th1/Th2 via targeting RUNX3 in psoriasis. Immunol Lett 166: 55-62, 2015.

30. Feng C, Bai M, Yu NZ, Wang XJ and Liu Z: MicroRNA-181b negatively regulates the proliferation of human epidermal keratinocytes in psoriasis through targeting TLR4. J Cell Mol Med 21: 278-285, 2017.

31. Yu X, An J, Hua Y, Li Z, Yan N, Fan W and Su C: MicroRNA-194 regulates keratinocyte proliferation and differentiation by targeting Grainyhead-like 2 in psoriasis. Pathol Res Pract 213: 89-97, 2017.

32. Lerman G, Avivi C,MardoukhC,Barzilai A, Tessone A, Gradus B Pavlotsky F, Barshack I, Polak-Charcon S, Orenstein A, et al: MiRNA expression in psoriatic skin: Reciprocal regulation of hsa-miR-99a and IGF-1R. PLoS One 6: e20916, 2011.

33. Li R, Qiao M, Zhao X, Yan J, Wang X and Sun Q: MiR-20a-3p regulates TGF- $\beta 1 /$ Survivin pathway to affect keratinocytes proliferation and apoptosis by targeting SFMBT1 in vitro. Cell Signal 49: 95-104, 2018

34. Zhu H, Hou L, Liu J and Li Z: MiR-217 is down-regulated in psoriasis and promotes keratinocyte differentiation via targeting GRHL2. Biochem Biophys Res Commun 471: 169-176, 2016.

35. Wang Y, Yu X, Wang L, Ma W and Sun Q: miR-320b is down-regulated in psoriasis and modulates keratinocyte proliferation by targeting AKT3. Inflammation 41: 2160-2170, 2018.

36. Ichihara A, Jinnin M, Yamane K, Fujisawa A, Sakai K, Masuguchi S, FukushimaS, Maruo K and Ihn H: microRNA-mediated keratinocyte hyperproliferation in psoriasis vulgaris. Br J Dermatol 165: 1003-1010, 2011.

37. Chowdhari S, Sardana K and Saini N: miR-4516, a microRNA downregulated in psoriasis inhibits keratinocyte motility by targeting fibronectin/integrin $\alpha 9$ signaling. Biochim Biophys Acta Mol Basis Dis 1863: 3142-3152, 2017.

38. Jiang M, Sun Z, Dang E, Li B, Fang H, Li J, Gao L, Zhang K and Wang G: TGF//SMAD/microRNA-486-3p signaling axis mediates keratin 17 expression and keratinocyte hyperproliferation in psoriasis. J Invest Dermatol 137: 2177-2186, 2017.

39. A R, Yu P, Hao S and Li Y: MiR-876-5p suppresses cell proliferation by targeting Angiopoietin-1 in the psoriasis. Biomed Pharmacother 103: 1163-1169, 2018.

40. Joyce CE, Zhou X, Xia J, Ryan C, Thrash B, Menter A, Zhang W and Bowcock AM: Deep sequencing of small RNAs from human skin reveals major alterations in the psoriasis miRNAome. Hum Mol Genet 20: 4025-4040, 2011.

41. Jiang M, Ma W, Gao Y, Jia K, Zhang Y, Liu H and Sun Q: IL-22-induced miR-122-5p promotes keratinocyte proliferation by targeting Sprouty2. Exp Dermatol 26: 368-374, 2017.

42. Xiong Y, Chen H, Liu L, Wang Z, Tian F and Zhao Y: microRNA-130a promotes human keratinocyte viability and migration and inhibits apoptosis through direct regulation of STK40-mediated $\mathrm{NF}-\kappa \mathrm{B}$ pathway and indirect regulation of SOX9-meditated JNK/MAPK pathway: A potential role in psoriasis. DNA Cell Biol 36: 219-226, 2017. 
43. Hermann H, Runnel T, Aab A, Baurecht H, Rodriguez E, Magilnick N, Urgard E, Šahmatova L, Prans E, Maslovskaja J, et al: miR-146b probably assists miRNA-146a in the suppression of keratinocyte proliferation and inflammatory responses in psoriasis. J Invest Dermatol 137: 1945-1954, 2017.

44. Luo Q, Zeng J, Li W, Lin L, Zhou X, Tian X, Liu W, Zhang L and Zhang X: Silencing of miR155 suppresses inflammatory responses in psoriasis through inflammasome NLRP3 regulation. Int J Mol Med 42: 1086-1095, 2018.

45. Zibert JR, Lovendorf MB, Litman T, Olsen J, Kaczkowski B and Skov L: MicroRNAs and potential target interactions in psoriasis. J Dermatol Sci 58: 177-185, 2010.

46. Wu R, Zeng J, Yuan J, Deng X, Huang Y, Chen L, Zhang P, Feng H, Liu Z, Wang Z, et al: MicroRNA-210 overexpression promotes psoriasis-like inflammation by inducing Th1 and Th17 cell differentiation. J Clin Invest 128: 2551-2568, 2018.

47. Guo S, Zhang W, Wei C, Wang L, Zhu G, Shi Q, Li S, Ge R, Li K Gao L, et al: Serum and skin levels of miR-369-3p in patients with psoriasis and their correlation with disease severity. Eur J Dermatol 23: 608-613, 2013.

48. Wang C, Zong J, Li Y, Wang X, Du W and Li L: MiR-744-3p regulates keratinocyte proliferation and differentiation via targeting KLLN in psoriasis. Exp Dermatol 28: 283-291, 2019.

49. Rachakonda TD, Schupp CW and Armstrong AW: Psoriasis prevalence among adults in the United States. J Am Acad Dermatol 70: 512-516, 2014.

50. Baliwag J, Barnes DH and Johnston A: Cytokines in psoriasis. Cytokine 73: 342-350, 2015.
51. Nograles KE and Krueger JG: Anti-cytokine therapies for psoriasis. Exp Cell Res 317: 1293-1300, 2011.

52. Wu GC, Pan HF, Leng RX, Wang DG, Li XP, Li XM and Ye DQ: Emerging role of long noncoding RNAs in autoimmune diseases. Autoimmun Rev 14: 798-805, 2015.

53. Tay Y, Rinn J and Pandolfi PP: The multilayered complexity of ceRNA crosstalk and competition. Nature 505: 344-352, 2014.

54. Li CX, Li HG, Huang LT, Kong YW, Chen FY, Liang JY, Yu H and Yao ZR: H19 lncRNA regulates keratinocyte differentiation by targeting miR-130b-3p. Cell Death Dis 8: e3174, 2017.

55. Akiyama M: Early-onset generalized pustular psoriasis is representative of autoinflammatory keratinization diseases. J Allergy Clin Immunol 143: 809-810, 2018.

56. Deng Y, Chang C and Lu Q: The inflammatory response in psoriasis: A Comprehensive review. Clin Rev Allergy Immunol 50: 377-389, 2016.

57. Lin X, Jiang T, Bai J, Li J, Wang T, Xiao J, Tian Y, Jin X, Shao T, $\mathrm{Xu}$ J, et al: Characterization of transcriptome transition associates long noncoding RNAs with glioma progression. Mol Ther Nucleic Acids 13: 620-632, 2018

58. Lei M, Mitsuhashi S, Miyake N, Ohta T, Liang D, Wu L and Matsumoto N: Translocation breakpoint disrupting the host SNHG14 gene but not coding genes or snoRNAs in typical Prader-Willi syndrome. J Hum Genet 64: 647-652, 2019.

(i) This work is licensed under a Creative Commons EY NO ND Attribution-NonCommercial-NoDerivatives 4.0 International (CC BY-NC-ND 4.0) License. 